Ans dem Zoologischen Institute der k. k. Deutschen Universität in rrag.

\title{
Zur Lichtentwicklung in den Photosphärien der Euphausien.
}

\author{
Von
}

Dr. Emanuel Trojan,

Assistenten am Zoologischen Institute der k. k. Deutschen Universität in Prag.

Hierzu 2 Textfiguren.

Im April 1906 hatte ich an der Zoologischen Station in Neapel während meines Aufenthaltes, den ich der Munifizenz der Gesellschaft zur Förderung Deutscher Wissenschaft, Kunst und Literatur in Böhmen verdanke, Gelegenheit, einen leuchtenden Schizopoden, Nyctiphanes Conchii in lebendem Zustande zu untersuchen. Da die Frage bezüglich der Lichtentwicklung in den Leuchtorganen des Tieres an lebenden Exemplaren nicht endgültig entschieden werden konnte, konservierte ich einiges Material. Die Bearbeitung desselben erfolgte im Zoologischen Institute der k. k. Deutschen Universität in Prag.

\section{Historischer Überblick.}

Unter den bis jetzt bekannten leuchtenden Krustern nehmen entschieden die Euphausien hinsichtlich des Baues ihrer Leuchtorgane den ersten Rang ein. Die Tatsache, dass diese Tiere Licht entwickeln, ist seit langem bekannt. Die erste sichere Angabe hierüber, abgesehen von dem schon viel früher bekannten, durch Sir Banks im 18. Jahrhundert gelegentlich seiner Weltumseglung entdeckten Leuchtvermögen mancher Schizopoden (wahrscheinlich auch Euphausien), finden wir bei D a n a (1852, S. 644) betreffend Euphausia splendens. Er schreibt: „Several individuals were observed to emit light, and it was seen to come from the anterior part of the cephalothorax. The light was intense and had a 'greenish tinge". Das Verdienst, den genauen Entstehungsort jenes Lichtes gefunden $\mathrm{zu}$ haben, gebührt aber erst Murray und Sars (1885, S. 70-72). Sars, der die Schizopoden der Challenger-Expedition bearbeitete, beschrieb als 
erster an den Euphausien die "luminous globules", dieselben Organe, die vor ihm ein einziger Forscher, nämlich Cl a u s (1863, S. 422) untersucht hatte. Claus sah in den vermeintlichen Or'ganen accessorische Augen und folgte darin der Ansicht Danas und Sempers (1862, S. 100); offenbar stand ihm nur totes Material zur Verfügung. Der Irrtum ist in diesem Falle verzeihlich, dazumal, wie Sars schon meint, der Bau dieser Leuchtorgane eine grosse Ähnlichkeit mit den Augen der Vertebraten hat. Weniger einleuchtend ist die Auffassung $\mathrm{Kr}$ o y e r s (1859, S. 294), der sie für Gehörorgane hielt. Übrigens weichen Claus und Sars nur bezüglich der Funktion der in Rede stehenden Organe voneinander $a b$, in der Beschreibung des anatomischen Baues derselben stimmen sie sonst überein. Beide finden, dass die Organe in kugeligen Auftreibungen der Körperdecke liegen, einen glänzenden Ring mit einer regelrechten Linse in der Mitte, dahinter, ungefähr im Zentrum des Organes, einen glänzenden aus zahlreichen Stäbchen bestehenden Körper, der in einer Zellmasse eingebettet ist, unterscheiden lassen. Diese ruht ihrerseits in einem halbkugeligen, vorne geöffineten Becher, dessen Aussenseite lebhaft rot pigmentiert ist, sodass die Organe trotz ihrer kleinen Dimension am Körper des Tieres leicht erkenntlich sind. Diese Beschreibung gilt nach Claus für die thorakalen und abdominalen Leuchtorgane. $\mathrm{S}$ a $\mathrm{r}$ s brachte es weiter, indem er erkannte, dass die Euphausien auch am Kopfe Leuchtorgane besitzen, die der Linse entbehren und sich auch sonst in der Form von den thorakalen und abdominalen wohl unterscheiden. $\mathrm{Claus}$ waren allerdings diese Organe keineswegs entgangen, denn bei dem Studium der Entwicklung von Euphausia (1863, S. 451) schreibt er wohl, dass er hinter den Augen anfangs "ein Bündel eigentümlich glänzender und eng aneinander liegender Stäbe " gesehen habe. Und von einem vorgeschrittenen Stadium lesen wir (l. c.): „Das bereits erwähnte Stäbchenbündel, dessen Axe zu der Längsaxe des Auges rechtwinklig nach unten gekehrt ist, liegt in orangegelbem Pigment von einem Rahmen umgeben, dessen Spitze wahrscheinlich zum Eintritte der Blutflüssigkeit durchbrochen ist". Trotz der grossen Ähnlichkeit, die diese Organe mit denen des Rumpfes zeigen, erkannte er sie als solche nicht und wusste daher über ihre Funktion noch weniger $\mathrm{zu}$ sagen, als über die jener, die er wenigstens als accessorische 
Augen ansah. Mets c b nikow (1869, S. 480), der die jüngsten Larvenstadien von Euphausien untersuchte, meinte, dass die von Claus beschriebenen, den Augen angehörenden Apparate "Nervenbüschel" seien. Murray (1885, Vol. I, S. 743) hat indessen auch diese Organe leuchten gesehen und Sars (l. c.) mit Recht unter die "luminous globules" eingereiht und als solche beschrieben.

Besondere Beachtung an den Leuchtorganen verdient nach seiner Meinung der "fibrous fascicle, lying in the centre of the globular corpuscle", denn er ist der Sitz des Lichtes. Wird er aus dem Zusammenhange des Organes gebracht, so leuchtet er eine Zeit lang weiter.

Es muss einen wundernelimen, dass, nachdem die Leuchtfunktion der Organe durch M urray und Sars erwiesen worden war, Patten im Jahre 1886 nach seinen Untersuchungen über die Augen der Arthropoden sich der alten Ansicht anschloss und die Leuchtorgane als Augen deutete. Ja vielmehr, ihm genügt es nicht mehr, sie Nebenaugen zu nennen, es sollen tatsächlich echte Augen sein, wie sie anderen Vertebraten zukommen. Dabei beschreibt er den Bau der Organe gar nicht eingehend und stellt überdies die einzelnen Teile ganz in den Dienst des Gesichtssinnes.

Diese irrige Auffassung seitens Patt en wurde durch $\mathrm{Pa}$ u $\mathrm{l}$ I a ye r und Wilh. Giesbrecht widerlegt. Da es ihnen geglïckt war, noch bevor $\mathrm{Pat}$ a $\mathrm{n}$ s Arbeit erschien, eine lebende Euphausie leuchten zu sehen, fügten sie seiner Publikation eine Anmerkung bei (1886, S. 738), in welcher die Angaben Sars eine Bestätigung erfahren.

Nicht anders handeln konnten Vallent in und Cunningham (1888), die den Schizopoden Nyctiphanes norvegica untersuchten. Diese Art besitzt auch Leuchtorgane, die den von Sars beschriebenen gleichen. Beide Autoren haben sie leuchten gesehen. Bezüglich des Sitzes der Lichtentwicklung in den Photosphärien aber sind sie einer anderen Meinung als Sars. Nicht die „fibrillar mass“ ("fibrous fascicle“ nach Sars) soll der Lichterzeuger sein, sondern jener äusserste, halbkugelige, auf seiner Aussenseite rot pigmentierte Becher, dem sie den Namen "Reflektor" geben. Ich glaube, dass diese Bezeichnung für die Vertretung der obigen Ansicht nicht zutreffend ist, denn niemals denkt man bei diesem Namen an die Selbsterzengung des Lichtes, sondern nur an ein 
Zurückwerfen des bereits vorhandenen. Immerhin traten diese beiden Autoren mit einer Ansicht auf, die seitdem nicht unberücksichtigt geblieben ist. Die Forscher, die sich mit den Leuchtorganen der Euphausien beschäftigten, teilten sich in zwei Lager: die einen - Sars, Chun (1896) und Giesbrecht (1896) hielten den Streifenkörper (fibrous fascicle), die anderen Vallentin und $\mathrm{Cu} n \mathrm{ningham}$ - den Reflektor für die Lichtquelle. Die Entscheidung, auf wessen Seite das Recht steht, ist noch nicht gefallen. Die vorliegende Mitteilung soll aber ein neues Licht auf die Sachlage werfen und uns der richtigen Beurteilung näher bringen.

\section{Die Leuchtorgane von Nyctiphanes Conchii.}

Der Bau der Leuchtorgane der Euphausien ist in vortrefflicher Weise bereits von $\mathrm{Ch} \mathrm{un} \mathrm{(1896)}$ geschildert worden. Meine diesbezüglichen Befunde an Nyctiphanes Conchii bestätigen, abgesehen von einigen Abweichungen, im grossen und ganzen die seinen.

Ich beginne mit den thorakalen, bezw. abdominalen Leuchtorganen. Es ist geboten, beide Arten unter einem zusammenzufassen, da sie dem inneren Baue nach vollkommen miteinander übereinstimmen. Dagegen müssen sie von denen des Kopfes wohl unterschieden werden. Es liegt je ein Paar am Grunde des zweiten und siebenten Thorakalfusspaares und vier unpaare ventral in der Mitte der vier ersten Abdominalsegmente, wie es bei den übrigen Angehörigen der Gattung Euphausia, Benteuphausia und Stilocheiron ausgenommen, der Fall ist. Diese, ihrer Form nach halbkugeligen Gebilde, haben verschiedene Grösse. Bei den thorakalen misst der grösste Breitendurchmesser $0,22 \mathrm{~mm}$, der grösste Höhendurchmesser $0,2 \mathrm{~mm}$; an den abdominalen ergibt die analoge Messung $0,15 \mathrm{~mm}$ und $0,16 \mathrm{~mm}$. Ch un machte bereits auf die leichte Beweglichkeit der Organe, die er für den Bau des Tiefenauges und für das Sehen in grossen Tiefen zu verwerten wusste, aufmerksam. Es ist unschwer, seine diesbezüglichen Angaben $\mathrm{zu}$ bestätigen. Eine vollkommene Serie streng geführter Querschnitte durch das ganze Tier lässt erkennen, dass das erste Leuchtorganpaar die Mündung unter einem Rechten, das zweite unter einem Winkel von ungefahr $45^{\circ}$ nach aussen kehrt; die abdominalen richten ihre Mündung stets nach unten. 
Aus diesem Grunde, sowie aus der Insertion von Muskeln hatte $\mathrm{Chun}$ wohl allen Grund anzunehmen, dass die ersten in einer zur Medianebene des Tieres unter $90^{\circ}$, die zweiten in einer unter $45^{0}$ geneigten Ebene, die letzten nur in der Medianebene beweglich sind. Deshalb gehört es auch zu den Seltenheiten, einen regelrechten Axialschnitt durch ein abdominales Leuchtorgan $\mathrm{zu}$

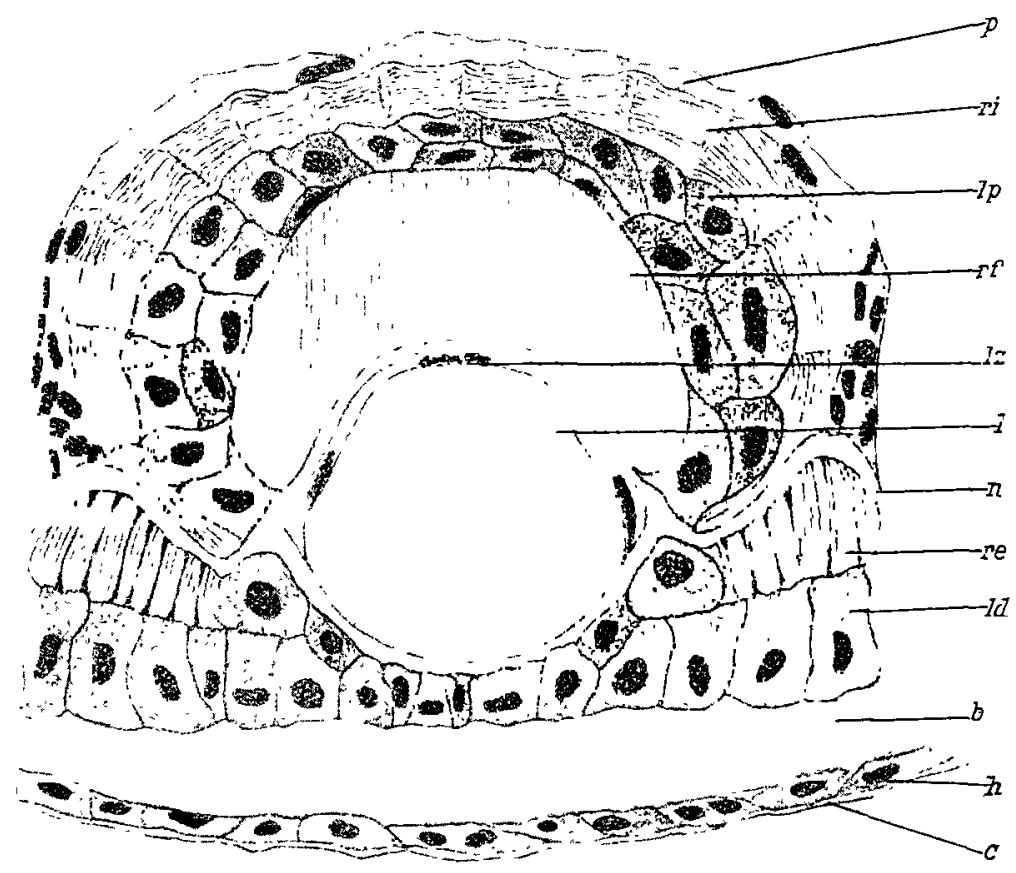

Fig. 1.

Ein thorakales Leuchtorgan von Nyctiphanes Conchii. Medianschnitt. $\mathrm{b}=$ Blutsinus, $\mathrm{c}=$ Cuticula, $\mathrm{h}=$ Hypodermis, $\mathrm{ld}=$ distale Leuchtzellen, $\mathrm{lp}=$ proximale Leuchtzellen, $\mathrm{l} z=$ Linzenzellen, $\mathrm{n}=\mathrm{Ner}$, re $=$ äusserer Reflektor, rf $=$ Refraktor, $r i=$ innerer Reflektor, $\mathrm{p}=$ Pigment. Vergr. 40 fach.

erlangen. Auf die Beweglichkeit des Organes weist übrigens auch der Umstand hin, dass es allseits von der Matrix der Chitinivand des Körpers losgelöst ist.

Die äusserste Hülle der Leuchtorgane bildet ein rotgefärbter Reflektor (r). Er besteht aus zwei Teilen, einem inneren becherförmigen und einem ausseren ringförmigen, der die ötfnung des ersteren im Kreise umzieht, in keinem Punkte aber mit ihm 
zusammentrifft, sondern durch eine Bindegewebslamelle getrenut ist. Dieser Teil entbehrt auch jeder Pigmentierung, was man von dem becherförmigen nicht sagen kann, da dieser überall von lebhaft roten Pigmentzellen (p) eingehüllt wird. Leider bewahren dieselben ihre Farbe in keinem Konservierungsmittel. Beide Teile sind aus konzentrischen Lamellen aufgebaut.

In der Darstellung dieser Reflektorschichten weiche ich von $\mathrm{Chun}$ etwas ab. Was zunächst den becherförmigen Teil anbelangt, so war es mir nie gelungen, jene glatten Lamellen ron grösserer Ausdehnung zu finden, die sich zu einer nahezu vollkommenen Hohlkugel zusammenlegen. Stets sah ich deutliche Grenzen zwischen Gruppen übereinander geschichteter Lamellen von beschränkten Dimensionen; ich habe dieselben auf meiner Zeichnung zum Ausdrucke gebracht. Dieser Umstand rief in mir den Gedanken wach, ob nicht zwischen den Lamellen und den darunterliegenden Zellen (Ip) eine Korrelation bestehe. Ich werde noch Gelegenheit haben, auf diese Sache zurückzukommen. Dass dieses Verhalten des inneren Reflektors auf Schrumpfung zurückzuführen wäre, halte ich für ausgeschlossen, da derselbe bei den Organen des Kopfes eine solche Eigentümlichkeit nie zeigte. In dem äusseren Reflektorring sah $\mathrm{Ch}$ u $\mathrm{l}$ an der Innenseite Bildungszellen und stellte die Vermutung auf, dass die Lamellen des Ringes aus diesen Zellen hervorgehen, und jener von seiner Innenseite an Dicke zunimmt, ,indem hier neue Lamellen den alten sich zugesellen unter allmählicher Rückbildung der zwischenliegenden sezernierenden Zellen". Vallentin und Cunningham sollen nach Angabe desselben Autors diese Zellen entgangen sein. Ich muss gestehen, dass ich mir alle Mühe nahm, um bei Nyctiphanes Conchii jene Zellen aufzufinden, doch umsonst. Dagegen fiel mir etwas anderes an jenem Ringe auf; die Lamellen desselben standen gruppenweise im engeren Zusammenhange miteinander, so dass, wie dort am becherförmigen Teile des Reflektors Gruppen von horizontal liegenden Schichten, hier solche von vertikal aufgerichteten zu sehen waren. Allerdings fïrbten sich die Spatien zwischen den einzelnen Bündeln stärker und täuschten einen Kern von länglich ovaler Gestalt vor; in der Tat war aber ein solcher nicht zu finden. Auch hier konnte ich es nicht unterlassen, die Gruppierung der reflektierenden Lamellen ohne Beziehung auf die benachbarten, auf der Aussenseite liegenden Zellen (ld) zu bringell. 
Ich wende mich nunmehr jener Gruppe von Zellen (lp, ld) $z \mathfrak{u}$, die ich soeben und oben bei der Beschreibung des inneren Reflektorteiles vorübergehend erwăhnt habe. Sie liegen überall dem Reflektor unmittelbar an, dem inneren Becher in zwei Lagen, dem ausseren in einfacher Schichte. Die letzteren scheinen bei ihrer Entwicklung mehr Raum gehabt zu haben und sind daher zylindrisch oder prismatisch. Den ersteren steht nur wenig Raum zu Gebote und daher mag wohl auch ihre unregelmässige, zumeist polyedrische Form herrühren. Auffällig gross sind die Kerne dieser Zellen, die manchmal den ganzen Raum innerhalb der Zellmembran ausfüllen. Ihr überaus leichtes Tinktionsvermögen lisst bequem die innere Granulation erkennen. Der plasmatische Teil der Zellen ist weniger gut tingierbar und macht auf mich mit seiner körnigen, bisweilen auch Vakuolen zeigenden Struktur den Eindruck, dass hier sezernierende Zellen vorliegen. Dies ist auch von früheren Autoren erkannt worden. Doch bezüglich des ausgeschiedenen Produktes gehen die Meinungen auseinander. Während Giesbrecht (1896, S. 490) diese Zellen als die Erzeuger des Leuchtsekretes ansieht, erfahren wir von $\mathrm{Cbun}$, „dass sie den Reflektor und den sogenannten Streifenkörper bilden" (1896, S. 201). Bevor ich nun die diesbezüglichen Resultate meiner Untersuchungen hier zum Ausdrucke bringe, erachte ich es für nötig, den Streifenkörper zu beschreiben. Doch eines möchte ich gleich hier bemerken. Es ist über jeden Zweifel erhaben, dass die Zellen (lp) innerbalb des becherförmigen Teiles identisch sind mit denen (ld), die dem atusseren Reflektor anliegen. Dafïr gibt weniger ihre Gestalt, als vielmehr ihr innerer Bau, das Aussehen der Kerne, das Verhalten gegen Farbstoffe ein sicheres Zeugnis. Warum sollte es dann besonderer Bildungszellen für den Reflektorring, die, wie oben erwähnt $\mathrm{Chun}$ an dessen Innenseite sah, bedürfen? Äusserer und innerer Teil des Reflektors sind von gleicher Zusammensetzung, und daher auch die sie abscheidenden Zellen von einerlei Art.

Und nun zu dem sogenannten Streifenkörper. Diese Bezeichnung rührt von $\mathrm{Chun}$ her. Er tauschte sie mit der alten - Stäbchenbündel nannte man ihn früher - ein, da er erkannte, dass diese für den Bau des besagten Gebildes weniger zutreffend sei; denn tatsächlich bestehe es aus Lamellen, die im Schnitte den Eindruck von Stäbchen hervorrufen, selbst aber solche nicht 
sind. Übrigens war aber die Bezeichnung „Stäbchenbündel“ nicht ungerechtfertigt, da Giesbrecht (1896, S. 488) bei Larven von Euphausien stets parallele Stäbchen an diesem Körper vorfand und niemals Lamellen; diese bilden sich vermutlich mit dem zunehmenden Alter des Tieres aus. Bei den thorakalen und abdominalen Leuchtorganen von Nyctiphanes Conchii sind sämtliche Lamellen vertikal, streng parallel geschichtet und bilden zusammen eine nach aussen offene hohle Halbkugel. Mag man den Schnitt durch das Organ führen wie man will, immer wieder tritt parallele Streifung an dem Gebilde zu Tage. Dies ptlegt im allgemeinen nicht vorzukommen, denn $\mathrm{Chun}$ spricht stets von "radiär ausstrahlenden" Lamellen und bildet solche auch ab. Er sieht in ihnen ein ungemein reizvolles System.

Ich habe dem Streifenkörper sowohl an lebendem Material, als auch an den durch das Leuchtorgan geführten Schnitten, besondere Aufmerksamkeit zugewendet und bin überzeugt, dass er derselben Natur ist wie der Reflektor, „ein totes Produkt, Sekret oder Cuticulargebilde" (Giesbrecht 1896, S. 488) der oben erwähnten sezernierenden Zellen. Seine Lamellen lösen sich bei frischem Material unter mechanischen Einflüssen ebenso in Fasern auf wie die des Reflektors; sie zeigen nach der Konservierung dieselbe Sprödigkeit wie jene, nur vielleicht noch in erhöhtem Maße. Gegen Farbstoffe verhalten sie sich gerade so, wie jene. Durch Pikrinsäure werden sie hochgelb gefärbt, andere Tinktionen nehmen sie weniger gut an.

Vor dem Streifenkörper, ungefähr bis zur Hülfte in ilı eingesenkt, liegt die von Dan a (1852, S. 644) bereits entdeckte Linse. Sie hat bei Nyctiphanes Conchii nahezu liugelform und ist homogen. Grosse, abgeplattete Kerne, die nach $\mathrm{Chun}$ ibren Bildungszellen angehören, liegen ihr dicht an.

Die Innervation der thorakalen und abdominalen Leuchtorgane bei dieser Art weicht von der, wie sie Chun (1896, S. 207) bei anderen Euphausien beschreibt, ab. Allerdings umgreifen auch hier zwei Äste des Nerven (n) in einem Bogen das Organ, um seitlich in dasselbe einzutreten. Die Stelle des Eintrittes befindet sich wie in der Regel zwischen dem inneren und äusseren Reflektor. Während aber $\mathrm{Cbun}$ den Verlauf des Nerven im Inneren des Leuchtorganes, soweit er sich verfolgen liess, so darstellt, als ob die Nerven sich dem Streifenkörper allein zu- 
kehren würden, sah ich an meinen Präparaten, dass jeder der beiden eingetretenen Äste sich zu den Seiten der Linse dichotom teilt und sie umgreift. Den weiteren Verlauf des Nerven habe ich nicht verfolgt, doch soviel steht sicher, dass die vor der Linse gelegene Partie nicht minderen Anteil an der Innervierung hat als die hinter ihr gelegene.

Die Leuchtorgane werden allseits von einem Blutsinus (b) umgeben.

Was nun jene am Kopfe gelegenen anbelangt, verweise ich

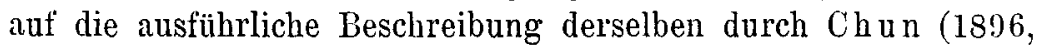
S. 199-203). Diese Leuchtorgane unterscheiden sich von thorakalen und abdominalen durch ihre konische Form, den dütenförmigen Streifenkörper, das Fehlen einer Linse und den terminalen Eintritt des Nerven. Übrigens habe ich den Streifenkörper bei Nyctiphanes Conchii grösser gefunden als ihn Chun bei Nematoscoelis rostrata darstellt. Sonst stimmen meine diesbezüglichen Befunde mit seinen überein.

\section{Funktion.}

Betreffs der Lichtentwicklung in den Leuchtorganen der Euphausiden habe ich bereits eingangs erwähnt, dass Sars und Chu n den Streifenkörper für die eigentliche Lichtquelle halten. Giesbrecht (1886, S. 499) schliesst sich dieser Meinung an; Vallentin und Cunningham teilen sie nicht. Welche Gründe bewogen die Forscher zu ihren Annahmen? S ars zerquetschte die Leuchtorgane lebender Schizopoden und sah allemal den Streifenkörper aufblitzen. Vallent in und Cunningham taten desgleichen, sahen jedoch den Streifenkörper niemals leuchten. Chun untersuchte lebendes Material ïberhaupt nicht und deshalb kann seine diesbezügliche Annahme nur als blosse Vermutung dahingestellt werden. Giesbrecht wollte der Sache von einer andern Seite beikommen und verfolgte die Entwicklung der Leuchtorgane. Es ist ihm nicht gelungen, die Frage endgültig zu lösen, doch insofern bedeutet seine Arbeit einen Fortschritt, als er mit Bestimmtheit dem Reflektor ein selbständiges Leuchtvermögen absprechen konnte; denn larvale Leuchtorgane sah er leuchten, bevor noch eine Spur vom Reflektor vorhanden war. Andererseits sollte er aber nicht behaupten, dass lediglich der Streifenkörper die eigentliche Lichtquelle sei, denn ausser diesem, 
wie er selbst schreibt, waren bereits Zellen ausgebildet, die ibn einschlossen und manchmal auch umhüllten. Dies können nur die sezernierenden Zellen (lp, ld) gewesen sein.

Meine Untersuchungen, die ich diesbezüglich an lebenden Nyctiphanes Conchii vornahm, gestalteten sich in folgender Weise.

Da die üblichen Vittel, die das Leuchten herbeiführen, wie mechanische Reize und Reagentien, Ammoniak, destilliertes Wasser (Brunnenwasser leistete dieselben Dienste) über die wahre Lichtquelle innerhalb des Organes keinen Aufschluss zu geben vermochten, wandte ich die alte Methode an und zerquetschte die Or'gane. Ich wusste, dass diese Art der Untersuchung als unverlässlich hingestellt wurde, $\mathrm{da}$ sie $\mathrm{zu}$ entgegengesetzten Resultaten (siehe oben Sars und Vallentin - Cunningham) gefübrt hat. Bei meinen Versuchen hat sie sich bewäht. Ich kann sagen, dass ich weder den Streifenkörper, noch weniger aber den Retlektor habe jemals leuchten gesehell. wofern ihm nicht eine Spur der sezernierenden Zellen (lp, ld) anhaftete und zwar nur eine Spur, denn in der 'Tat waren es oft gar keine Zellen, sondern bloss Überreste von solchen, der gekörnte Plasmaschleim. Umgekehrt aber leuchteten niemals Stellen unter dem Deckglase auf, wo ein Bestandteil des Retlektors, oder ein solcher vom Streifenkörper nachzuweisen war. Dafür machten aber die Lamellen dieser beiden Gebilde den Eindruck voll stark lichtbrechenden Medien, namentlich die des letzteren in erhöhtem Grade. Und so bin ich zu der Überzeugung gelangt, dass die wah re Lichtquelle in den Leuchtorganen der Euphausidien die sezernierenden proximalen. beziehungsweise peripheren Zellen (lp, ld), die ich daher Leuchtzellen nenne, sind. In ihnen kommt das Leuchtsekret zustande. Ihr granuliertes Plasma erinnert mich in seinem tinktoriellen Verhalten an das jener Zellen in den Leuchtorganen der Fische, die als Lichterzeuger betrachtet werden mussten, da sich sonst im Leuchtorgan nichts anderes vorfand, dem diese Funktion luatte zugeschrieben werden können. Hier liegen die Sachen offenbar so. Das Leuchtsekret ist zugleich der Baustoff sowohl für den Reflektor, als auch für den Streifenkorper; es leuclitet beim Austritt aus der Zelle, in der es bereitet worden ist; durch mechanischen Reiz (Zerquetschen des Organes) kann es früher zum Leuchten gebracht werden. So wird es erklärlich, warum Sars den Streifenkörper, 
Vallentin-Cunningham den Reflektor leuchten sahen. Wären ferner diese beiden Gebilde ihrer Natur nach verschieden,

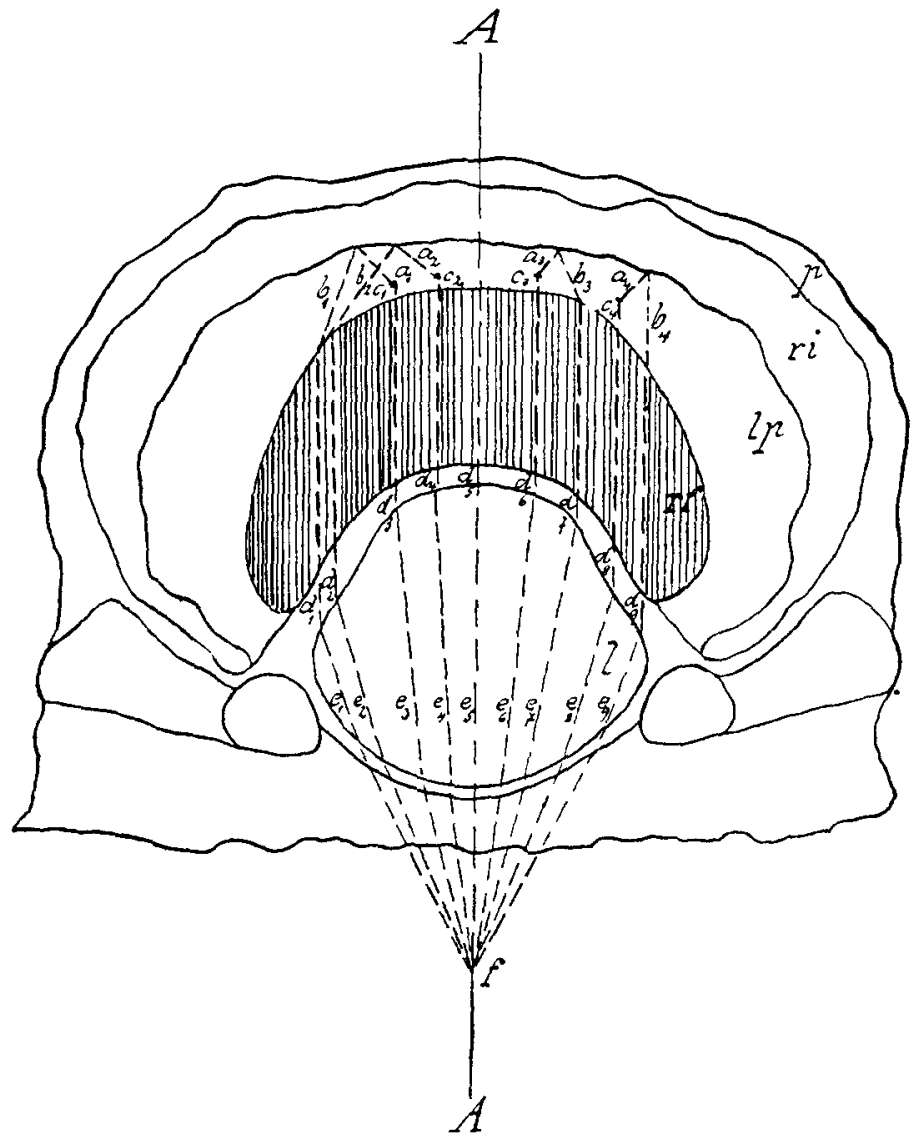

Fig. $\supseteq$.

Schematischer Axialschnitt durch ein thorakales Leuclitorgan von Nyctiphanes Conchii : $a 1-a t, b 1-b+$ Lichtstrahlen, die in der Zone der proximalen Leuchtzellen entstehen und rom inneren Reflektor (ri) zurickgeworfen werden; c1-c \& Lichtstrahlen, die den direkten Weg nach aussen nehmen; d1-d9 Lichtstrahten nach dem Durchgange durch den Refraktor (rf); e 1-e9 Lichtstrahlen, durch die Linse (1) gebrochen; $\mathrm{A}=$ Achse, $\mathrm{f}=$ Brennpunkt, $\mathrm{l}=$ Linse, $\mathrm{lp}=$ proximale Leuchtzellen, $\mathrm{rf}=$ Refralitor; $\mathrm{ri}=$ innerer Reflektor, $\mathrm{p}=$ Pigment. Verg1. 40) fach.

verstünde man wohl schwer, wie jene Zellen (lp), die bei manchen Arten nur in einschichtiger Lage angetroffen werden, an ihrem 
distalen Ende etwas anderes absondern sollten als an ihrem proximalen.

Es erübrigt nunmehr noch die Frage, welche Rolle spielt der Streifenkörper beim Leuchten? Die hohe Lichtbrechung und sein innerer Bau weisen darauf hin, dass er beim Leuchtprozess als Refraktor ausgezeichnete Dienste leisten muss. Die Strahlen, die in seinem Umkreise entstehen, müssen eine bestimmte Richtung annehmen. Die hier beigefügte Konstruktion macht die Sache leicht verständlich.

Man sieht, dass nahezu kein Lichtstrahl aus dem becherförmigen Teile des Organes heraustreten kann, ohıe seinen Weg durch den Refraktor genommen zu haben. Dass bei manchen Arten die Lamellen nicht parallel sind, sondern konisch zusammenlaufen, das widerspricht meiner Auffassung nicht im geringsten, im Gegenteil ist ihr nur förderlich. Sollte aber ein Einwand erhoben werden, dass meiner Ansicht nach Leuchtzellen auch ausserhalb des becherförmigen Teiles und zwar die dem ringförmigen anliegenden (ld) angetroffen werden und dass Lichtstrahlen aus diesen Zellen unmöglich durch den Refraktor gehen können, gebe ich dies vollkommen $z u$; diese Strahlen sind eben nur auf den Reflektor angewiesen.

So kann ich am Schlusse meiner Betrachtung zusammenfassen, dass in den Leuchtorganen der Schizopoden die distalen und proximalen Leuchtzellen die Quelle des Lichtes sind; Linse, Refraktor (Streifenkörper) und Reflektor sind bloss optische Hilfsapparate.

Prag, im Februar 1907. 


\section{Literaturverzeichnis.}

1896 Chun, C.: Leuchtorgane und Facettenatgen. Atlantis. Bibl. Zool. Heft 19, S. 193-209, Taf. 16-20.

1863 Claus, C.: Über einige Schizopoden und Malakostraken Messinas. Zeitschr. f. wissenschaftl. Zool., Bd. 13, S. 422, Taf. 25-29.

$182^{2}$ D a n a, J. D.: United States Exploring Expedition. Vol. XIII, Crustacea. Philadelphia, 69 Tafeln.

1896 Giesbrecht, W.: Über den Sitz der Lichtentwicklung in den Photosphärien der Euphausiiden. Zool. Anz., Jahrg. XIX, S. $486-490$.

1859 Kroyer, H.: Forsög til en monographisk Fremstilling af Kraebsdyrslaegten Sergestes. Kong. Danske Videnskab. Selsk. Skrifter, Bd. 4 , S. 219-303, 5 Tafeln.

1886 Mayer-Giesbrecht: Mitt. Zool. Stat. Neapel, Bd. 6, S. 756.

1869 Metschnikow, El.: Über ein Larvenstadium von Euphausia. Zeitschrift f. wissensch. Zool, Bd. XIX, S. 479-483, Taf. XXXVI.

1885 Muray, J.: The voyage of H. M. S. Challenger. Narrative of the Cruise.

1886 Patten, W.: Eyes of Molluses and Arthropods. Mitt. Zool. Stat. Neapel, Bd. 6, S. 542-756, Taf. $28-32$.

1885 Sars, G. O.: The voyage of H. M. S. Challenger. Report on the Schizopoda. Vol. XIII, 38 Tafeln.

1862 Semper, K.: Reisebericht. Zeitschr. f. wissenschaft1. Zool., Bd. 11, S. $100-108$.

1888 Valentin, R. und Cunningham, J. T.: The Photospheria of Nyctiphanes Norvegica G. O. Sars, Quarterly Journal of Microscopical Science, Bd. XXVIII, S. 318-341, Taf. 23. 